\title{
A COMPARATIVE STUDY OF THE VARIABILITY OF BOYS AND GIRLS
}

By Grorge W. Frasier, Washıngton State Normal School, Cheney.

During the past decade much has been written and said about the variability of the sexes. One school of psychology maintains that there are no median sex differences of mental capacities, but the ability of the male is 'scattered over a greater range, that is, he is more variable. They thus account for the fact that more men than women have become famous, also that more men are in the low levels of society. The other school of psychology believes that there are no sex differences either in variability or central tendency. Furthermore they maintain that it is the present constitution of society that puts men into the extremes. The question of variability as applied to children is of vast practical importance to those who deal with boys and girls Shall we deal with children on the assumption that boys should be found in the extremes and the girls in the middle ground?

The literature of the subject can be separated into two classes (1) studies dealing with men and women and making no mention of boys and girls and (2) those dealing with children. It is evident that many students of this subject draw their data from the behavior of adults, make their conclusions on such data and then apply their findings in dealing with children This attitude is not justified by facts. The following study deals with school children, and for this reason the quotations taken from other authors will be confined to those dealing with boys and girls.

Keyes made a special study of all the children in one school district, having six or more annual records available. He found 1,902 children distributed as is shown in Table I.

TABLE 1

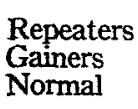

Total ..

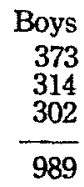

Girls

310

299

304

913 
He concludes from the above table 1 "The boys in this group (repeaters) are more numerous than the girls, as would be expected from the known greater variability of the male," also "The gainers or accelerates were 613 in number; 314 of them boys, 299 girls. The greater variability of boys is here again shown."

A casual glance at the table seems to vindicate the conclusion made by Keyes. However, it is to be noted that the totals show 76 fewer girls than boys. Now, Keyes has assumed that had these 76 girls been present all of them would have been in the "Normal" group. To overcome this inequality and show the true comparison between the boys and girls it is necessary to reduce each group to the percentage of the total. The percentage grouping is shown in table 2 .

TABLE 2

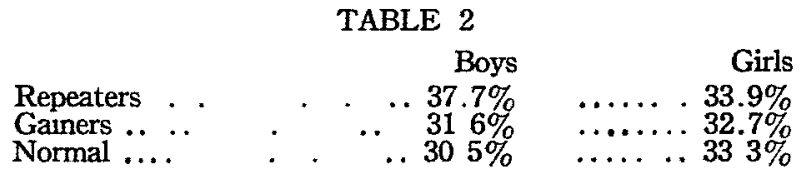

The above table indicates that when these data are reduced to a comparable basis the greater variability of the boys vanishes.

Thorndike believes that boys are more variable than girls, for he writes " "Athough the male and female types are closely alike in intellectual capacities, there is an important difference in the deviations from the type in the two cases, namely, that the males deviate more The highest males in any quality are more gifted than any of the women, and the lowest males inferior to all women. Thus, though girls in general rank as high or higher than boys in high school and college, they less often lead the class; thus there are far more eminent intellects among men than among women and twice as many idiots." In a foot-note he offers the following explanation: "This difference is not absolutely proven to exist but it appears fairly certain from the results of several investigations."

Rogers and McIntire in measuring the intelligence of children with the Binet-Simon scale found that ${ }^{3}$ "The principal outcome is the familiar law that there is a wider scattering of ability among boys than among girls; consequently there are more gifted as well as more backward boys than girls," and

${ }^{1}$ C. $\mathrm{H}$ Keyes Progress through the Grades of Ctty Schools, p. 15, 17 and 32.

2 E. L Thorndike. Principles of Teaching, p. 96.

${ }^{3}$ Britush Journal of Psychology, Igr4, vol $7, \mathrm{p} 265$ 
" so far as we go, our experiments support the general law, which several observers have already noted, that there is a greater variability of capacities in boys than in girls, but no general superiority."

The most complete study made in America dealing with the case in point was made by Terman. Terman studied 1,000 unselected school children from the standpoint of intelligence and came to the conclusion that " The supposed wider variation of boys is not found. Girls do not group themselves about the median more closely than do boys. The range of I $Q$ including the middle fifty per cent is approximately the same for the two sexes."

Leta Hollingworth made a very complete resumé of studies concerned with the variability of the sexes and pointed out that up to 1914 these had not established the greater variability claimed for the males. She says "-and there is at the present time no conclusive empirical evidence to show that in cases where the coefficient of variation is greater for one sex than for the other, this greater variability consists of greater range." 5

It appears from the quotations given that the subject is far from settled, and for this reason the following data are presented.

It is claimed by the school of variability that whenever males and females, or boys and girls, undertake a task that the best progress and the slowest progress will be made by the boys, and the girls will compose the great bulk of median ability. Then, one would expect this law to hold true in school accomplishment.

It appears that should we select all of the boys and girls of a certain age in any school system or systems we could, by finding their grade location, determine the relative variability of boys and girls of that age.

Thirteen was selected as a desirable age because (1) children of this age are held in school by the attendance laws in most states and (2) at 13 a child has been in school long enough to demonstrate his ability.

The grade location of all thirteen-year-old boys and girls was found for twenty cities: ${ }^{6}$ Boston, Mass.; Columbus, Ohio; Wichita, Kansas; Grand Rapids, Mich.; Kansas City, Mo.; Richmond, Va.; Winston-Salem, N. C.; Baltimore, Md.;

\footnotetext{
${ }^{4} \mathrm{~L}$ M. Terman. The Measurement of Intelligence, p. 70

5 Americait Journal of Sociology, 1914, vol. 19, p. 510-530

B The data concerning these children were taken from the city school reports
} 
Cleveland, Ohio; Trenton, N. J.; Philadelphia, Pa.; Danbury, Conn.; Fall River, Mass ; Winchester, Mass.; Beverly, Mass.; York, $\mathrm{Pa}$; Williamsport, Pa ; Aurora, Ill.; St. Louis, Mo.; Waterloo, Iowa. Table 3 gives the total number of each sex in each grade.

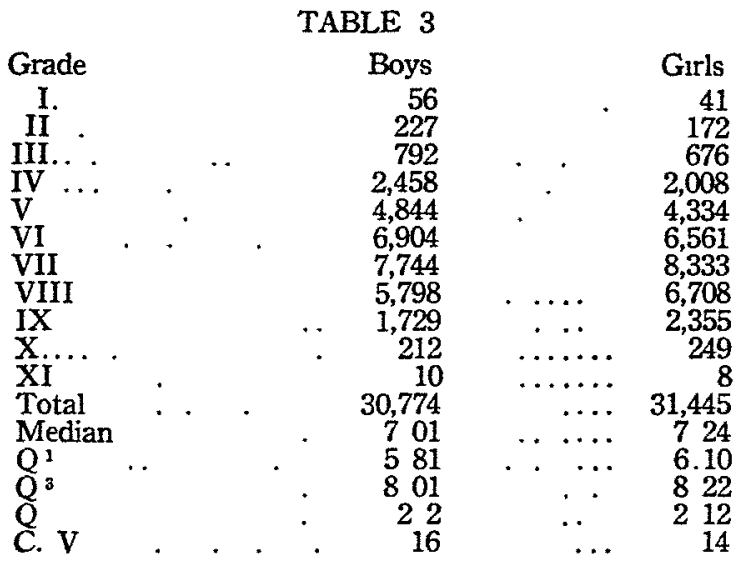

The coefficient of variability for the boys is slightly greater than that for the girls but the difference is so very small that no greater variability can be said to exist. In order to show all possible comparisons between the boys and girls chart $A$ is given showing nine different percentiles, for each sex and their relative locations.

It is evident from the chart presented that girls make better school progress than boys. From the standpoint of variability our data point clearly to the conclusion that no sex differences are shown. The statistical procedure is carried out from the second to the ninety-eighth percentile so that the question of greater range is disposed of.

It is safe to conclude from the study of 62,219 thirteenyear-old boys and girls in 20 cities that the greater variability claimed for the boys is not present. It appears that this greater variability has never been established with any large number of cases or with any degree of certainty and if it is to be used as a working basis for those dealing with boys and girls it will be necessary for its supporters to present some conclusive empirical evidence. Until this is done it seems safest to disregard such a theory and deal with boys and girls on the supposition that they are alike so far as mental variability is concerned. 


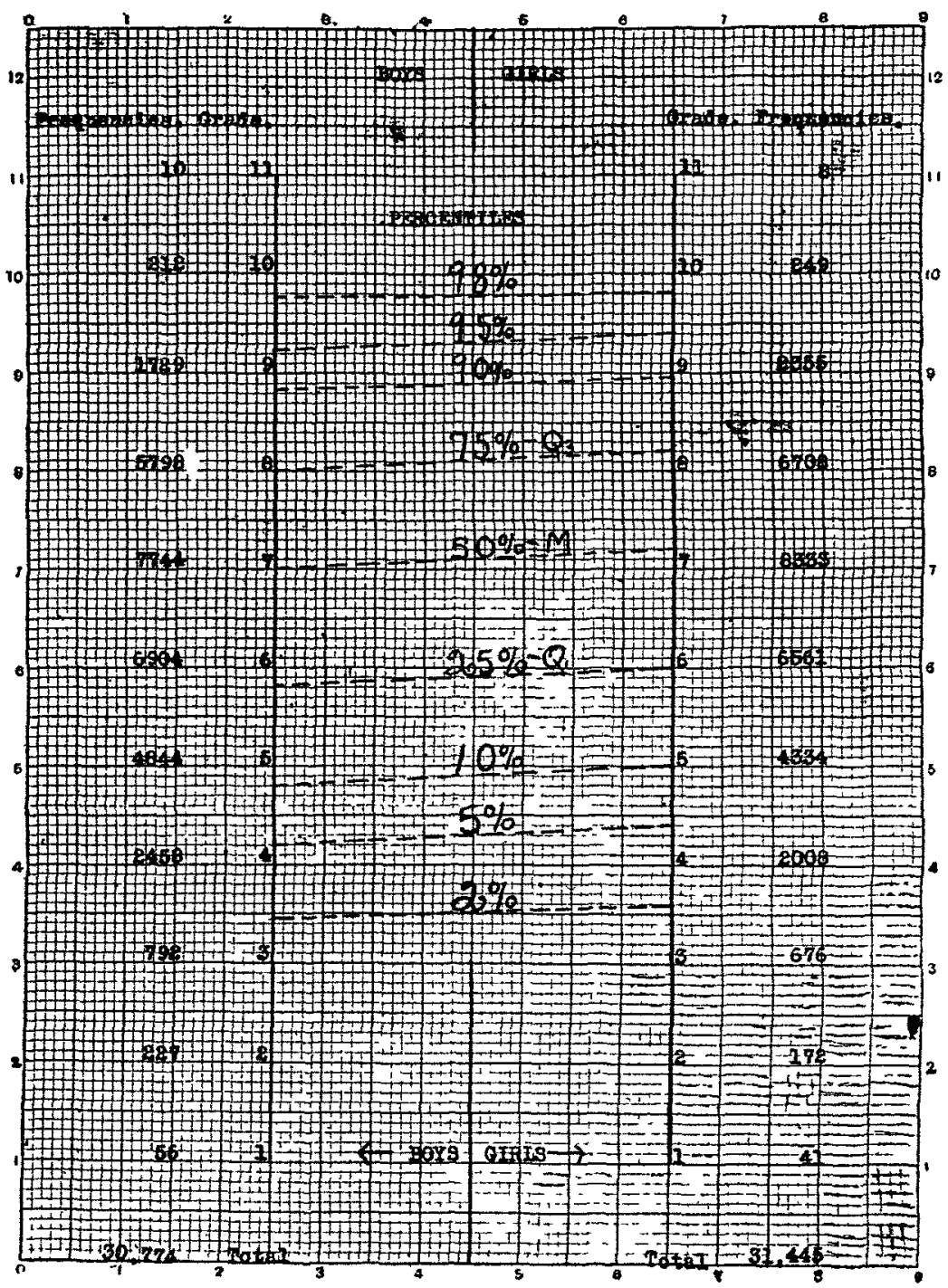

